\title{
Emerging Monoclonal Antibody Therapy for the Treatment of Acute Lymphoblastic Leukemia
}

\author{
Tareq Abuasab ${ }^{1}$ \\ Jacob Rowe ${ }^{1-3}$ \\ Ariella Tvito'
}

'Department of Hematology, Shaare Zedek Medical Center, Jerusalem, Israel; ${ }^{2}$ Department of Hematology and Bone Marrow Transplantation, Rambam Health Care Campus, Haifa, Israel; ${ }^{3}$ Bruce Rappaport Faculty of Medicine, Technion, Israel Institute of Technology, Haifa, Israel
Correspondence: Jacob Rowe

$\mathrm{Tel}+972-502063199$

Fax + 972-26555755

Email rowe@jimmy.harvard.edu

\begin{abstract}
The treatment of adults with ALL has undergone tremendous progress over the past 15 years. The advances have been particularly marked with B-lineage ALL. The development of bispecific antibodies directed against CD19 ushered in a new era in overcoming persistent minimal disease in newly diagnosed ALL patients as well as successfully treating those with relapsed disease. The immune-conjugates targeting CD22 have also had a similarly impressive role in improving the outcome in such patients. These advances are now being extended to frontline regimens for B-lineage ALL, including the Philadelphiachromosome-positive subtype. Over the past decade, the development of chimeric antigen receptor T-cell therapy (CAR-T) has ushered in a new era, opening up hope when none was available for patients with particularly advanced disease. Such advances come at a considerable price for toxicity, which, however, are lessening with experience and the development of new agents to ameliorate some of the toxicities. Unfortunately, the progress for T-cell in ALL has lagged behind that of B-lineage ALL. Of late, however, there are preliminary results of potentially exciting data using monoclonal antibodies against CD38, in the form of daratumumab, and it is hoped that these will lead to an equally successful advance in the treatment of T-ALL. Despite all these advances, ALL in adults remains a formidable disease. While ongoing progress is being made, also in the therapy of older patients, we are still lagging behind the almost totally curative potential of current therapy for childhood ALL.
\end{abstract}

Keywords: acute lymphoblastic leukemia, monoclonal antibodies, blinatumumab, inotuzumab ozogamicin, rituximab

\section{Introduction}

Unlike pediatric acute lymphoblastic leukemia (ALL), which is curable in $>90 \%$ of cases, adult ALL including older patients still has a dismal prognosis with overall cure rates of less than $40 \%{ }^{1}$. This discrepancy is mainly due to the different genomic landscapes and poorer tolerability of adults to prolonged intensified chemotherapy. B-cell ALL accounts for approximately $75 \%$ of all ALL cases and has historically been associated with an inferior outcome compared with T-cell ALL. Historically, the therapy of ALL was based on a backbone of chemotherapy which included induction, intensification and prolonged maintenance for two to three years. This template is generally used for B- and T-cell lineage ALL. Relapsed disease was treated with chemotherapy and, depending on donor availability, with an allogeneic stem cell transplantation. Over the recent years, major advances in understanding the biology and pathophysiology of the disease have allowed for the identification of molecular targets and markedly expanded treatment options, 
particularly for B-cell ALL, including novel antibody constructs, tyrosine kinase inhibitors (TKIs) targeting the BCR-ABL1 and chimeric antigen receptor (CAR-T) cell therapy. ${ }^{2}$ Many clinical trials have been initiated to investigate the best treatment strategy and optimize the inclusion of these novel therapies, both in advanced and in early stages of the disease. Improved genetic profiling and increased sensitivity of immunophenotyping have led to greater dependence on minimal measurable disease (MRD) for prognostication and guidance of therapy. Targeted agents, mostly in the form of directed antibodies, have altered the landscape of ALL such that dramatic advances have been made in the treatment of patients with residual MRD after induction, as well as those in relapse. This review will focus on major aspects of the emerging monoclonal antibody therapy and their integration in the treatment of adult ALL.

\section{Monoclonal Antibodies}

\section{Rituximab}

CD20 is a B-cell differentiation antigen. It is a non-glycosylated transmembrane phosphoprotein that has an important role in cell cycle progression, differentiation and modulation of apoptosis pathways. ${ }^{3} \mathrm{CD} 20$ is expressed on approximately $30-50 \%$ of the precursor B-cell lymphoblasts. ${ }^{4}$

Rituximab, a chimeric monoclonal antibody against CD20, has prolonged the survival of patients with CD20positive non-Hodgkin lymphoma as well as Burkitt Lymphoma. ${ }^{5}$ These encouraging results led to randomized studies investigating the addition of rituximab to standard chemotherapy in CD20+ ALL patients.

In a prospective trial at the MD Anderson Cancer Center in Houston, USA, for patients with precursor B-cell ALL, 12 doses of rituximab were added to the standard treatment with hyper-CVAD. In the younger CD20-positive patients (age $<60$ years), adding rituximab improved complete remission (CR) duration ( $67 \%$ vs $49 \%$; $\mathrm{p}<0.002)$ and overall survival (OS), $(75 \%$ vs $47 \%, \mathrm{p}=0.003)$. Older patients, though, did not benefit from rituximab-based chemoimmunotherapy (OS $28 \%$ vs $32 \%, \mathrm{p}=\mathrm{NS}$ ). This was possibly attributed to the high rate of myelosuppression-related deaths in this group. ${ }^{6}$

The German ALL study group evaluated the incorporation of rituximab into conventional chemotherapy in 263 Philadelphia chromosome ( $\mathrm{Ph}$ )-negative $\mathrm{CD} 20$-positive precursor B-cell ALL patients aged $15-55$ years. ${ }^{7}$ Eight doses of rituximab were added to induction, re-induction and consolidation of the standard regimen. There was no difference in the CR rate after induction ( $94 \%$ vs $93 \%$ ), but the rate of MRD negativity was superior at day 24 (60\% vs $19 \%)$ and at week $16(90 \%$ vs $59 \%)$. In addition, the 5 -year CR rate $(80 \%$ vs $47 \%)$ and OS ( $71 \%$ vs $57 \%)$ were significantly higher.

In a pivotal study, the prospective, randomized, Phase III trial by the French Group for Research in Adult ALL (GRAALL), ${ }^{8}$ the addition of rituximab was evaluated in the pediatric-inspired GRAALL2005 protocol for patients aged 18-59 years with newly diagnosed CD20positive Ph-negative B-Cell Precursor (BCP)-ALL. A total of 16 to 18 rituximab infusions were administered during induction, re-induction, consolidation, late intensification and maintenance. The CR rate was similar in the rituximab and control arm (92\% vs 91\%). However, with a median follow-up of 30 months, patients treated in the rituximab arm had a lower cumulative incidence of relapse ( $34 \%$ vs $20 \%, \mathrm{P}=0.029)$ and exhibited improvement in the two-year event-free survival (EFS) $(65 \%$ vs 52\%, p=0.038) (Figure 1) and OS rates $(71 \%$ vs $64 \%, p=0.095$; with censoring for HSCT, $\mathrm{p}=0.018)$.

Infectious events were slightly more frequent in the rituximab group; the difference though was not significant.

Taken together, these trials show the significant benefit of adding rituximab to standard chemotherapy regimens, particularly in young patients ( $<60$ years). Accordingly, the addition of rituximab to multi-agent chemotherapy has become the standard of care for younger ( $<60$ years) patients with newly diagnosed CD20+ B-cell ALL. Although there is no definitive evidence that adding rituximab in older CD20+ B ALL patients improves outcomes, it seems reasonable to incorporate rituximab into the therapeutic backbone due to the favorable safety profile of rituximab.

\section{Ofatumumab}

Ofatumumab is a second generation, fully humanized monoclonal antibody against $\mathrm{CD} 20$. It binds to a different extracellular epitope of CD20 with a greater avidity than rituximab, which promotes a more complement-dependent cytotoxicity. ${ }^{9}$

Ofatumumab was evaluated in a Phase II trial in combination with hyper-CVAD in newly diagnosed $\mathrm{Ph}$ negative B-cell ALL patients with any level of CD20 expression (ie, $\geq 1 \%$ ). ${ }^{10}$ 


\section{Rituximab for B-lineage, CD20+, ALL}

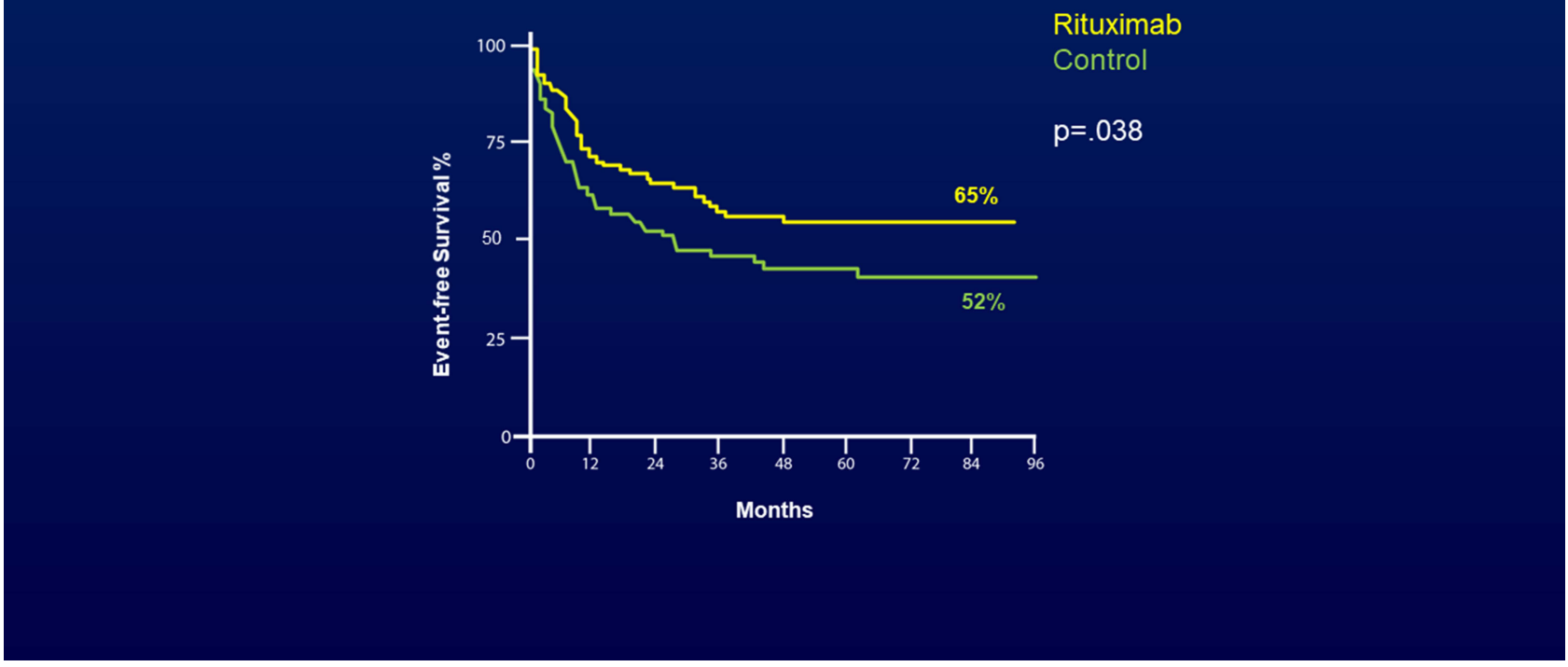

Figure I Prospective GRAALL-R 2005 study of addition of rituximab for patients aged 18-59 years with newly diagnosed CD20-positive Ph-negative B-cell precursor (BCP)-ALL. Rituximab was administered during induction, re-induction, consolidation, late intensification and maintenance. The improvement in the 2 -year EFS (65\% vs $52 \%$, $\mathrm{p}=0.04)$ at 2 years and ( $55 \%$ vs $43 \%$ ) at 4 years is shown in this figure. From The New England Journal of Medicine, Maury S, Chevert S, Thomas X, et al, Rituximab in Blineage adult acute lymphoblastic leukemia, 375(II), 1044-1053. Copyright @ (2016) Massachusetts Medical Society. Reprinted with permission from Massachusetts Medical Society. ${ }^{8}$

The overall response rate was $98 \%$, with a 4 -year event-free survival of $59 \%$ and 4 -year OS of $68 \%$. The clinical benefit was seen across all CD20+ subgroups ( $>20 \%$ and below $20 \%$ ). Thus, adding ofatumumab to chemotherapy seems a reasonable option especially in patients with low levels of CD20 $(<20 \%)$ where rituximab has not been studied. However, a direct comparison of the safety and efficacy of ofatumumab with rituximab is not possible due to its non-randomized single-arm design. In addition, it is not known whether ofatumumab improves long-term outcomes compared with rituximab. In practice, given the incorporation of rituximab into the standard of care, following the data from the GRAALL2005 study, ofatumumab is infrequently used.

\section{Epratuzumab}

CD22 is expressed on leukemic blasts in more than $98 \%$ of patients with B-lineage ALL. ${ }^{11}$ Epratuzumab is a fully humanized monoclonal antibody directed against CD22, and upon its binding, it leads to a rapid internalization of the antibody/antigen complex making it a promising therapeutic target in B-cell ALL. Its mechanism of action includes antibody-dependent cellular cytotoxicity, CD22 phosphorylation and proliferation inhibition with crosslinking. ${ }^{12}$

Pediatric data from the ADVL04P2 study by the Children's Oncology Group ${ }^{13}$ reported that adding epratuzumab to the standard chemotherapy improved the rate of MRD negativity (39\% vs $25 \%$ ) with no increase in toxicity and without any impact on the overall survival.

In adult ALL, a phase II study by the Southwest Oncology Group (SWOG) evaluated epratuzumab combined with clofarabine plus cytarabine in 31 patients with CD22-positive relapsed disease. ${ }^{14}$ The response rate was significantly higher than in a previous trial using the same chemotherapeutic regimen alone (52\% vs $17 \%)$. Given these data, the IntReALL (International study for treatment of childhood Relapsed ALL), initiated a randomized phase III trial (NCT01802814) testing the effect of epratuzumab in relapsed pediatric patients that was intended to shed light on the potential role of this antibody in the treatment of relapsed ALL. Unfortunately, the randomization was terminated prematurely in February 2019 (due to lack of drug availability), and it is not known whether the 
curtailed study will be sufficient to provide data that can be confidently interpreted. This would be unfortunate as its low toxicity profile with an almost universal presence in B-ALL, makes this naked antibody an attractive agent for this common subtype of ALL.

\section{Daratumumab}

Immunotherapy for T-ALL has been more challenging, especially due to difficulties of identifying surface antigens uniquely expressed on leukemic blasts but not on normal T-cells. Recent advances have been made in this field, mainly with daratumumab. CD38 is highly expressed on the surface of $\mathrm{T}$ and B ALL blasts, ${ }^{15}$ which makes it an ideal target. ${ }^{16}$ Daratumumab is an anti-CD38 monoclonal antibody, known to be safe and effective in treating relapsed refractory multiple myeloma. The main side effects include infusion reactions, which are mostly mild. Additionally, daratumumab interferes with blood group serological testing, as it binds to $\mathrm{CD} 38$ on red blood cells and results in a positive indirect Coombs test.

Daratumumab has multiple mechanisms of action, which simultaneously target tumor cells and the tumor microenvironment, including complement-dependent cytotoxicity (CDC), antibody-dependent cellular cytotoxicity (ADCC), antibody-dependent cellular phagocytosis (ADCP), programmed cell death, modulation of enzymatic activity, and immunomodulatory activity. ${ }^{17}$

Daratumumab was tested in a large panel of T-ALL patient-derived xenografts and found to have a striking efficacy in 14 of 15 different xenografts, suggesting daratumumab to be a promising novel therapy for T-ALL patients. ${ }^{18}$ These data were confirmed in several patients with relapsed T-ALL. One case report describes an adult T-ALL patient in her third relapse after two prior allogeneic transplants. ${ }^{19}$ Following salvage therapy 15.6\% blasts were detected by flow cytometry. Daratumumab was initiated. After the third dose, she achieved MRD negativity. The patient completed 12 doses of daratumumab and continued to be in remission six months after initiation of treatment.

Another report described a newly diagnosed patient with T-ALL. He received daratumumab for postinduction refractory disease. After two cycles, he achieved a morphologic CR, but was MRD-positive. After further eight doses, he became MRD-negative. ${ }^{16}$

Daratumumab was also successfully administered for compassionate use in three patients with very high-risk advanced CD38-positive ALL relapse. ${ }^{20}$ Two patients had T-ALL, the third CD19/CD22-negative pre-B-ALL. All of them achieved MRD-negative remission after daratumumab with prolonged disease-free survival (10 and 10.5 months).

These promising reports led to an open-label, multicenter, phase II study (NCT 03384654) led by Jansen Research Development, LLC, evaluating the efficacy of daratumumab in addition to standard chemotherapy in pediatric participants with relapsed/refractory B-cell acute lymphoblastic leukemia (ALL)/lymphoblastic lymphoma (LL) and T-cell ALL/LL. This study is currently actively recruiting.

In summary, daratumumab, with its good response and low toxicity, is considered a potentially exciting therapy for relapsed/refractory CD38-positive ALL, particularly for T-lineage ALL given the current paucity of novel agent for this subtype of ALL If, in large prospective studies, efficacy will be confirmed in advanced T-cell ALL, it is likely in time to lead to trials incorporating this into the newly diagnosed setting - particularly in the high-risk subtypes such early precursor (ETP) T-ALL. In fact, such a trial is currently planned by the Alliance for Clinical Trials in Oncology of the National Cancer Institute (NCI).

\section{Alemtuzumab}

Alemtuzumab is a humanized monoclonal antibody against CD52, a glycoprotein found in $36-66 \%$ of leukemia cases including B/T ALL and AML. Alemtuzumab induces cell lysis through antibody dependent cellmediated cytotoxicity. ${ }^{21}$

The Children's Oncology Group (COG) conducted a limited institution phase II trial in 13 patients with relapsed or refractory ALL using alemtuzumab as a single agent. ${ }^{22}$ Only one of the 13 patients enrolled had a complete response and four had stable disease. In this trial, the efficacy of alemtuzumab's efficacy was limited, and it does not seem suitable as a single agent therapy.

In 2009, the Cancer and Leukemia Group B (CALGB 10102) conducted a Phase I trial with 24 CD52 positive ALL patients. ${ }^{23}$ They received alemtuzumab during postremission therapy with a median OS of 55 months and a median DFS of 53 months. However, alemtuzumab was associated with an increased risk of neutropenia and CMV viremia. 
Thus, due to its toxicity and only modest efficacy, the role of alemtuzumab in the treatment of relapsed/refractory ALL remains limited.

\section{Anti-IL-7 Monoclonal Antibodies}

Interleukin 7 (IL-7) is a cytokine produced in the thymus and bone marrow, and its receptor (IL-7R) is fundamental in the development of normal T cells. However, high IL-7/ IL-7R expression can also play a significant role in leukemogenesis and has been shown in vivo to accelerate human T-ALL development. ${ }^{24}$ It promotes the T-ALL cell cycle progression and viability by activating oncogenic pathways such as PI3K/Akt/mTOR and JAK STAT. $^{25}$ Leukemic blasts from the majority of T-ALL patients $(\sim 70 \%)$ display IL-7R and thus an attractive target in these patients. Monoclonal antibodies against IL$7 \mathrm{R} \alpha$ are being developed ${ }^{26}$ and, though not yet studied in a clinical setting, they seem promising.

\section{Bispecific Antibodies}

CD19 is a B lymphocyte cell surface marker expressed on normal and malignant $B$ cells. Its vast expression on the blasts of patients with B-cell precursor ALL makes it an ideal target for immunotherapy.

The purpose of immunotherapy is to activate the patient's own anticancer immunity in order to allow it to generate sustained cytolytic activity against malignant cells with the hope to completely eradicate the neoplastic process.

\section{Blinatumomab}

Blinatumomab is a bispecific $\mathrm{T}$ cell engager (BiTE). It combines two antigen-binding sites, one specific for $\mathrm{CD} 3$ cytotoxic T cells and the second specific for CD19 expressing leukemic cells, joined by a non-immunogenic linker. The simultaneous binding of blinatumomab to the B-ALL blast cells and the cytotoxic $\mathrm{T}$ cells brings the two cells in

\section{Blinatumumab Mechanism}

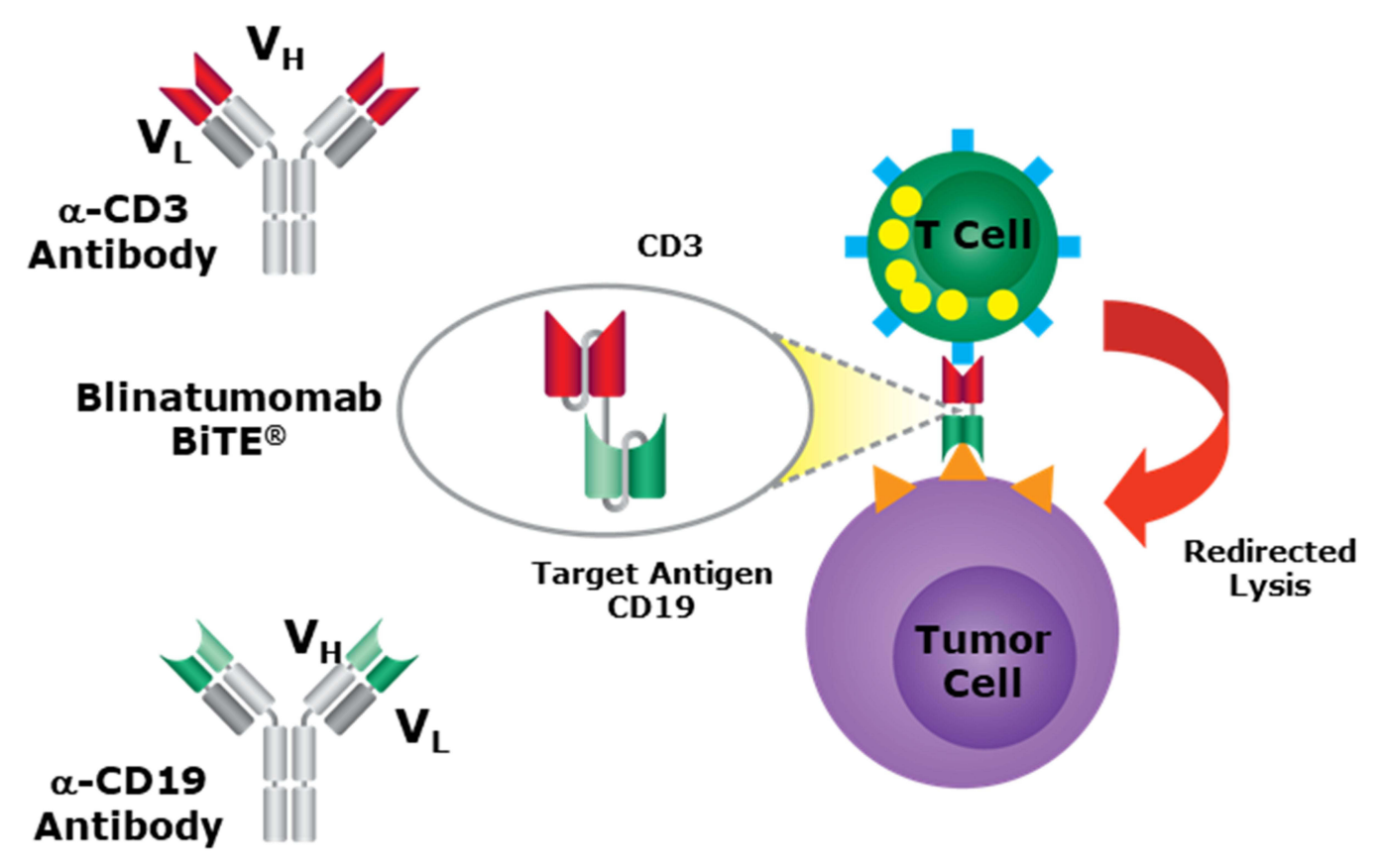

\section{- Blinatumomab is a bispecific T-cell engager antibody designed to direct cytotoxic T-cells to CD19 expressing cancer cells}

Figure 2 Figure showing the mechanism of action of blinatumomab, a bispecific single-chain antibody construct with dual specificity for CDI9 and CD3. The simultaneous binding of blinatumomab to the B-ALL blast cells and the cytotoxic T cells brings the two cells in close proximity and induces T-cell activation with subsequent cytotoxicity directed against CDI9 positive cells. From Bargou Bargou R, Leo E, Zugmaier G, et al. Tumor regression in cancer patients by very low doses of a T cell engaging antibody. Science. 2008;32I(589I):974-977. doi:10.1 I26/science. I I58545. Reprinted with permission from AAAS. ${ }^{27}$ 
close proximity and induces T-cell activation with subsequent cytotoxicity directed against CD19 positive cells ${ }^{27}$ (Figure 2).

Blinatumomab was highly efficient in phase I/II studies in relapsed/refractory B-cell ALL, particularly in the setting of lower disease burden. ${ }^{28}$ Minimal residual disease (MRD) is the strongest predictor of outcome in ALL. The German group conducted a phase II trial to evaluate whether blinatumomab as a single agent has the ability to eliminate MRD in patients with MRD-positive B-cell ALL. ${ }^{29}$ Among the 20 evaluated patients, 16 patients (80\%) became MRD negative. All MRD conversions were observed after only one cycle of treatment. $61 \%$ of these patients stayed relapse free after a median follow up of 33 months.

The most common side effects were fever, chills, headache, fatigue, peripheral edema and lymphopenia. These side effects can be partially explained by the mechanism of blinatumomab, which induces a polyclonal T-cell activation. The flu-like symptoms are caused by cytokine release and rapid rise in activated T-cells after starting the blinatumomab infusion.

Although most patients only suffer mild flu-like symptoms, some develop severe cytokine release syndrome (CRS) also known as macrophage-activation syndrome, an acute life-threatening systemic inflammatory syndrome characterized by fever and multi organ dysfunction. The pathophysiology of CRS is incompletely understood. Activated T cells release interferon gamma, which induces activation of macrophages. These produce excessive amounts of additional cytokines, especially interleukin- $6 .^{30}$ Thus, treatment with the interleukin-6 inhibitor tocilizumab may reverse this life-threatening condition. Based on insights from this trial, a prophylactic protocol consisting of cytoreduction, dose adjustment and premedication with corticosteroids has been devised, resulting in a reduced incidence of severe CRS. ${ }^{31}$

CRS prophylaxis with tocilizumab has also been studied with encouraging data. Caimi et al retrospectively studied the prophylactic role of tocilizumab in preventing CRS in lymphoma patients and concluded that the use of prophylactic tocilizumab (PT) reduced the incidence of severe CRS. In addition, importantly, this decreased rate of CRS was not associated with impaired disease control. $^{32}$

A large multicenter phase II study evaluated blinatumomab in 189 adult ALL patients with primary refractory, early relapsed disease or relapsed/refractory ALL after the first salvage therapy or beyond. ${ }^{28}$ In the final analysis, $30 \%$ of the patients achieved CR (30\%) and $10 \%$ reached a CRh (CR with incomplete hematologic recovery). Among the patients who achieved CR or CRh, 40\% subsequently underwent allogeneic hematopoietic stem cell transplant (HSCT). Considering the poor prognostic factors of this group of patients, these results were extremely impressive, and despite relying on historic controls, the data reported in this study led to the regulatory approval of blinatumomab by the Food and Drug Administration (FDA) in the USA (Figure 3). Approval in Europe, Japan and the rest of the world followed soon after.

The phase III multicenter TOWER study assigned heavily pretreated B-cell precursor ALL patients randomly to receive blinatumomab or standard care chemotherapy. ${ }^{33}$ Treatment with blinatumomab resulted in a significantly longer OS than chemotherapy (7.7 months vs 4 months; $\mathrm{P}=0.01)$, higher $\mathrm{CR}$ rates $(34 \%$ vs $16 \%, \mathrm{P}<0.001)$ and MRD negativity ( $76 \%$ vs $48 \%$ ). The main side effects were neurologic symptoms and cytokine release syndrome, which were severe in $10 \%$ and $5 \%$ of case, respectively. This trial highlights the potential of targeted therapy for patients with relapsed or refractory B-cell precursor ALL to reduce the need for prolonged, intensive chemotherapy.

The Italian Gruppo Italiano Malattie Ematologiche dell'Adulto (GIMEMA) reported recently the efficacy of blinatumomab in patients with a newly diagnosed $\mathrm{Ph}+$ B-cell ALL. ${ }^{34}$ A chemotherapy-free treatment consisting of TKI and blinatumomab led to a CR rate of $98 \%$, with $60 \%$ of patients reporting a negative MRD after two cycles of blinatumomab and dasatinib. DFS and OS at 18 months were $88 \%$ and $95 \%$.

There are two recent and ongoing phase III intergroup studies in newly diagnosed ALL patients led by the ECOGACRIN in the US. One randomized phase III trial (EA1910) evaluated treatment of chemotherapy with or without blinatumomab in newly diagnosed Ph-neg B-ALL patients (Figure 4). This study completed its accrual and if positive will lead to a change in the standard of care of newly diagnosed B-lineage ALL. The other ongoing randomized phase III trial for adults with newly diagnosed $\mathrm{Ph}+\mathrm{ALL}$ (EA9181), after a short prephase, prospectivel randomizes patients to either receive induction with a non-chemotherapy arm consisting of steroids, tyrosine kinase inhibitor (TKI) and blinatumomab versus standard chemotherapy. Patients who achieve MRD-negativity will receive TKI as maintenance with or without an allogeneic HSCT (investigator 


\section{Blinatumomab \& Relapsed/Refractory ALL}

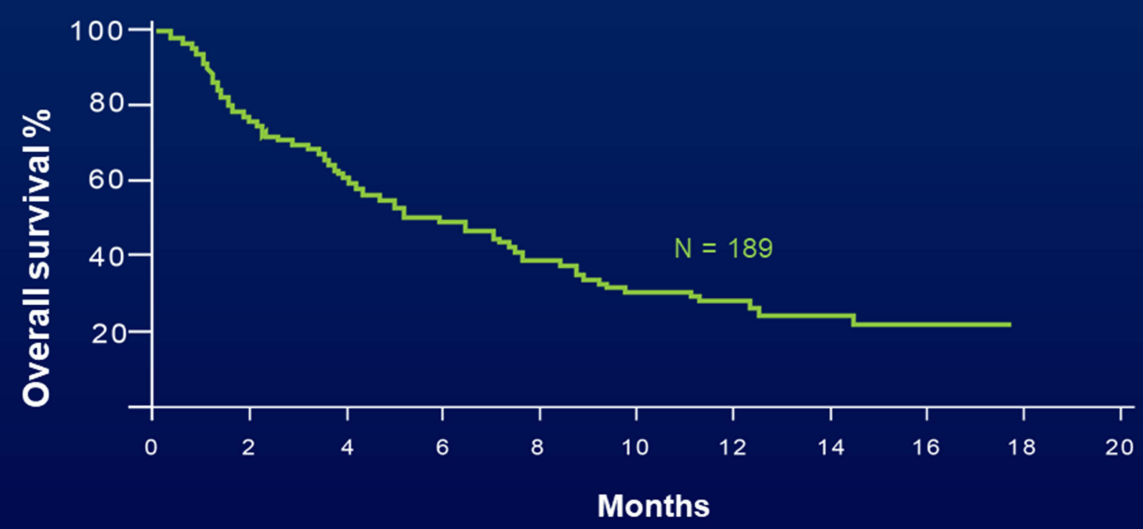

Figure 3 Blinatumomab in adult patients with primary refractory or relapsed Philadelphia chromosome-negative leukemia. A multicenter, single-arm, open-label phase II study of blinatumomab ( $9 \mu \mathrm{g} / \mathrm{day}$ for the first 7 days and $28 \mu \mathrm{g} /$ day thereafter) by continuous intravenous infusion over 4 weeks every 6 weeks (up to five cycles). Median overall survival was 6.1 months for all 189 patients, with a median follow-up of 9.8 months and relative odds ratio for survival benefit of achieving remission being 0.13 . Reprinted from The Lancet Oncology, 32(37), Topp MS, Gokburger N, Stein AS, et al., Phase II trial of the anti-CDI9 bispecific T cell-engager blinatumomab shows hematologic and molecular remissions in patients with relapsed or refractory B-precursor acute lymphoblastic leukemia, 4I34-4I40., Copyright 20I4, with permission from Elsevier. $^{28}$

\section{Intergroup study: ECOG-E1910: Phase III study of Blinatumomab in ALL}

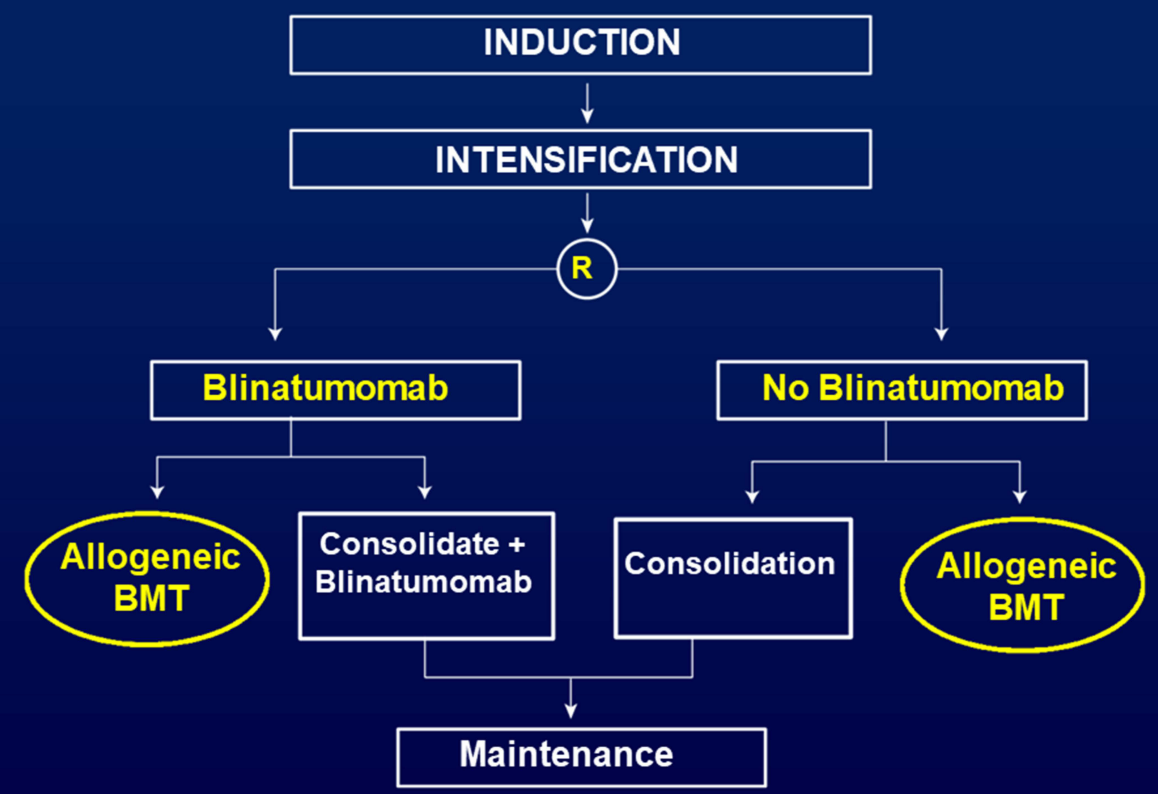

Figure 4 ECOG-ACRIN EI9I0 - Randomized phase III trial, now completed accrual, evaluating the impact of the addition of blinatumomab to newly diagnosed BCR-ABLnegative B-ALL. Although the initial CR is not impacted in this study, the focus is on increasing the depth of MRD-negativity and its potential to increase the long-term survival. 


\section{ECOG-ACRIN Study (EA9181) for Ph-positive ALL}

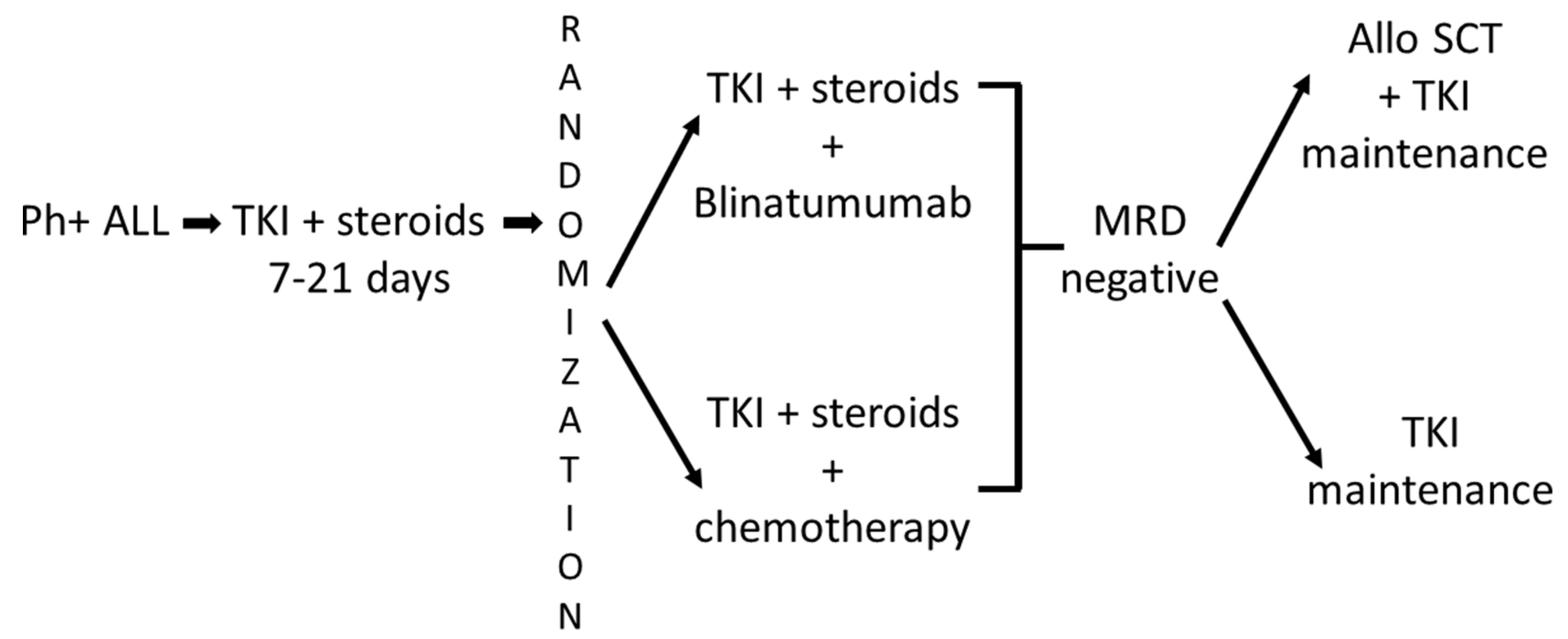

Figure 5 ECOG-ACRIN EA9I8I - An ongoing prospective phase III trial for newly diagnosed Ph+ ALL. After a short prephase of steroids and TKI, patients are randomized to receive two cycles of a non-chemotherapy arm consisting of steroids, TKI and blinatumomab versus a standard steroid, TKI and chemotherapy arm. Patients who remain MRD-positive can crossover the induction arms. Those who achieve MRD-negativity will receive TKI maintenance, with or without an allogeneic HSCT (investigator choice).

choice) (Figure 5). This trial, a registration study for the FDA, will definitively determine whether Ph-positive ALL patients can be successfully treated in the current era with a curative regimen that does not include any chemotherapy.

\section{Conjugated Antibodies}

Conjugated antibodies are antibody-drug conjugates (ADC). A monoclonal antibody, chemically linked to a cytotoxic agent, binds to an antigen on the cell surface of malignant cells, enabling delivery of the cytotoxic agent specifically to the tumor cell. This is not immunotherapy; rather, this is a targeted mechanism that facilitates increased efficacy with decreased toxicity.

\section{Inotuzumab Ozogamicin}

CD22 is a cell-surface glycoprotein expressed on most B cells. It is not expressed on hematopoietic stem cells or cells outside the hematopoietic system and is thus an attractive therapeutic target for B-cell cancers. Inotuzumab ozogamicin is an immune-conjugate consisting of anti-CD22 monoclonal antibody that is linked to calicheamicin, a DNA-alkylating agent. Encouraging results from phase II trials led to a multi-center phase III trial, known as the INO-VATE ALL trial. ${ }^{35}, 218$ patients with relapsed/refractory ALL were treated with either inotuzumab ozogamicin or standard therapy. Inotuzumab ozogamicin had a significantly higher CR rate $(80.7 \%$ versus $29.4 \%, \mathrm{p}<0.001)$. More patients in the inotuzumab group were able to proceed to stem cell transplantation $(41 \%$ versus $11 \%, \mathrm{p}<0.001)$. The median duration of remission and the progression-free survival (PFS) were also significantly longer. Median OS was 4.6 months versus 3.1 months, and the PFS was 5.0 months versus 1.8 months, respectively. These results were confirmed in a longer follow-up showing two-year OS rates of $23 \%$ with inotuzumab ozogamicin versus $10 \%$ with standard chemotherapy ${ }^{36}$ (Figure 6). The main adverse events of inotuzumab ozogamicin were hepatotoxicity including veno-occlusive disease (VOD). VOD rates were $11 \%$ versus $1 \%$ in the standard therapy group, mostly after HSCT and with use of dual-alkylator conditioning. Hematologic and infectious complications were lower than in standard chemotherapy.

These encouraging results led to clinical trials combining inotuzumab ozogamicin with low-intensity chemotherapy to further improve outcomes. In a study from the MD Anderson Cancer Center, 59 relapsed/refractory ALL patients were treated with inotuzumab ozogamicin and mini-H-CVD, a low-intensity version of the hyper-CVAD regimen, without doxorubicin. ${ }^{37}$ The overall response rate was $78 \%$, with $59 \%$ of the patients reaching CR with an MRD negativity rate of $82 \%$. Almost half of the patients underwent HSCT. The median relapse-free survival and 


\section{Inotuzumab Ozogamicin vs. Standard Therapy}

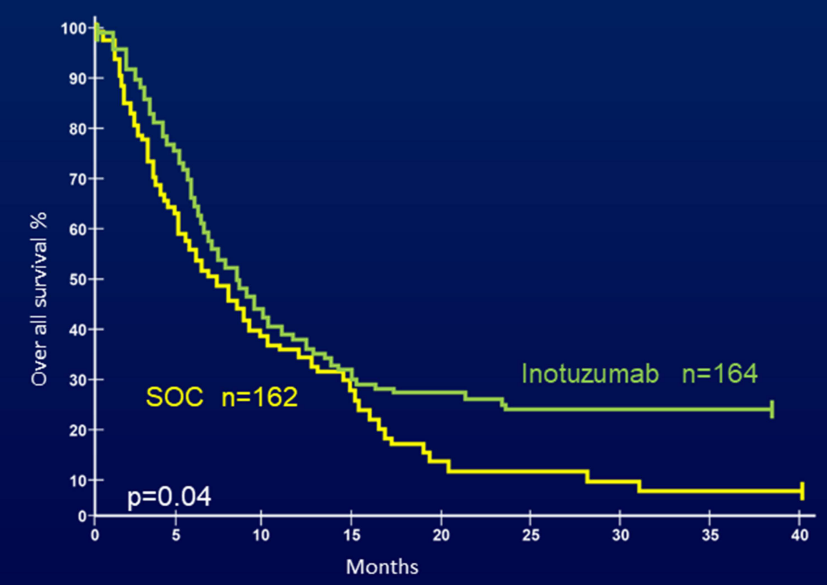

Figure 6 Figure showing the overall survival rate taken from INO-VATE trial where patients were randomized either to inotuzumab ozogamicin or standard of care therapy. The inotuzumab ozogamicin arm had a $25 \%$ lower risk of death than those in the standard of care arm with median OS 7.7 months vs 6.2 months. Exploratory post -hoc analysis at 2 and 3 years showed a larger OS rate difference between the treatment arms at later time points with 2 -year OS rates of $22.8 \%$ and $10.0 \%$ for the respective groups. Copyright @2019. John Wiley and Sons. Reproduced from Inotuzumab ozogamincin versus standard of care in relapsed or refractory acute lymphoblastic leukemia: final report and long-term survival follow-up from the randomized, Phase 3 INO-VATE study. Cancer. 2019;125(I4):2474-2487. doi:10.1002/cncr.32II6. ${ }^{36}$

OS were eight and 11 months. VOD occurred in $15 \%$, mainly in patients with prior or subsequent HSCT. The results were most encouraging in the first salvage, with a median OS of 17 months compared with nine months in the historical series treated without inotuzumab ozogamicin. This study has been further amended by adding four cycles of blinatumomab following 4 cycles of inotuzumab ozogamicin and mini-HCVD. ${ }^{38}$ This strategy might lead to higher response rates, lower treatment-related toxicity and improve long-term outcomes.

Another important area where inotuzumab ozogamicin may be used clinically is in the upfront treatment of ALL in an attempt to reduce the dose of intensive chemotherapy, such as the Hyper-CVAD. Older patients with newly diagnosed acute lymphoblastic leukemia have a poor outcome, with an estimated median overall survival of five to ten months, partially due to death during induction or remission when treated with intensive chemotherapy. In a phase II trial, 52 elderly patients with newly diagnosed Philadelphiachromosome-negative ALL were treated with inotuzumab ozogamicin combined with mini-hyperCVAD. ${ }^{39}$ The two-year progression-free survival was $59 \%$. The safety profile was tolerable, with no treatment-related deaths observed during induction therapy.

Given these data, the Alliance for Clinical Trials in Oncology, through the National Cancer Institute (NCI) in the USA, initiated a randomized phase III study (NCT03150693) evaluating the role of incorporating inotuzumab ozogamicin into frontline therapy of adolescents and young Adults (ages 18-39 years) with newly diagnosed precursor B-cell ALL.

\section{BCL-2 Inhibitor Venetoclax}

Venetoclax, a highly selective B-cell lymphoma 2 (BCL2) inhibitor, is an anti-apoptotic protein conferring enhanced survival in leukemia cells. Venetoclax is a safe and effective treatment for chronic lymphocytic leukemia and acute myeloid leukemia. ${ }^{40,41}$

Early precursor T-ALL (ETP) ALL is a distinct and aggressive subtype of T-ALL with low response to 
therapy, higher rates of post-induction MRD positivity, and inferior survival compared with non-ETP ALL. ${ }^{42}$ Cells from the ETP ALL subtype are known to be sensitive to BCL2 inhibition, suggesting that venetoclax might be an effective drug for the treatment of this subtype. ${ }^{43}$ A retrospective review at the MD Anderson Cancer Center evaluated the efficacy and safety of venetoclax in 13 patients with relapsed and refractory T-ALL treated with venetoclax and chemotherapy. ${ }^{44}$ Five patients $(38 \%)$ had early T-cell precursor ALL. Venetoclax was combined with various agents, including hyper-CVAD, asparaginase, nelarabine, decitabine, or other intensive chemotherapy. Six patients $(60 \%)$ achieved a remission with bone marrow blasts $<5 \%$, including three with a complete hematologic recovery. The median OS and RFS were 7.7 and 4.0 months, respectively.

Another Phase I, multicenter, open-label study was conducted to examine the safety and efficacy of venetoclax in combination with navitoclax (a BCL2 inhibitor that also inhibits BCLXL and BCLW) in patients with B-cell ALL, T-cell ALL, or lymphocytic lymphoma. ${ }^{45}$ The overall response rate (ORR) was $56 \%(20 / 36)$ in the total population.

In summary, BCL-2 inhibition appears to be an exciting new therapeutic approach as a single agent or in combination with other chemotherapies in patients with refractory/relapsed early precursor T-ALL.

\section{CAR-T Cell Therapy}

Chimeric antigen receptor (CAR)-T cells are patient's or donor's $\mathrm{T}$ cell, which are genetically engineered to combine an extracellular antigen recognition domain (CD19) with one or more intracellular $\mathrm{T}$ cell signaling domains (CD3). Second- and third-generation CAR T cells have increasing number of $\mathrm{T}$ cell receptor co-stimulatory domains, such as CD27, CD28, CD134 (OX40), or CD137 (4-1BB). T cells are collected from the subject before undergoing ex-vivo manipulation and expansion. They are transduced with a viral vector resulting in CAR cells with the ability to recognize specific cells independent of the major histocompatibility complex. Prior to their infusion, patients typically receive chemotherapy in an effort to induce lymphodepletion to enhance CAR T-cell expansion and persistence in vivo. The chimeric $\mathrm{T}$ cells are then returned to the patient in order to enable binding of their antigen-binding domain to leukemic CD19 receptor to exert direct cytotoxicity. ${ }^{46,47}$
CAR-T cell therapy has its own unique toxicity; it is associated with cytokine release syndrome (CRS) in $76 \%$ to $100 \%$ of the patients. ${ }^{48}$ Patients at high risk for severe CRS include those with large tumor burdens, with comorbidities, and patients who develop early onset CRS, within three days of infusion. ${ }^{49}$ Pathophysiologically, CRS is triggered by the activation of $\mathrm{T}$ cells when their CARs engage the designated antigens on the malignant cells. The activation leads to proliferation of CAR T cells and release of cytokines and chemokines from antigen-redirected T cells including IL-6, soluble IL-6 receptor, soluble IL-2 receptor a, interferon gamma (IFN- $\gamma$ ), and granulocytemacrophage colony-stimulating factor. ${ }^{50}$

In ALL, the disease burden prior to CAR-T infusion is a strong predictor for the severity of CRS. In addition, infusion dose and peak in vivo expansion correlate with the severity of CRS. ${ }^{46,51}$ Treatment of CRS was successfully done with a steroid and IL-6 monoclonal antibody tocilizumab with some data showing that steroids can decrease CAR-T cell level, while tocilizumab does not. ${ }^{48,52,53}$ Another potentially fatal CAR-T cells toxicity is neurologic, occurring in around $43 \%$ of patients, and can include encephalopathy, visual hallucinations, aphasia, delirium and seizures. Neurologic symptoms appear to be self-limited and related to inflammation rather than to CAR-T cells in the CNS themselves. Other CAR-T cell toxicities include grade B cell aplasia and severe cytopenia in most patients. In addition, hypokalemia, hypophosphatemia and liver function elevation were reported frequently. ${ }^{48}$

Many different anti-CD19 CAR-T cell therapies were used across different clinical trial programs, despite the differences in cellular products and clinical trial design (including the design of the CAR, the method of CAR transfer into $\mathrm{T}$ cells and the use of lentiviral vs retroviral vectors), aspects of the CAR-T-cell culture environment and the selection of CAR-T cells for infusion. Initial outcomes using anti-CD19 CAR-Ts across these Phase II trials for ALL are quite similar. High remission rates of $67-93 \%$ with minimal residual disease negativity was observed in adult and pediatric patients with heavily pretreated R/R ALL.

The first CAR T cell therapy approved by the FDA was tisagenlecleucel, an autologous anti-CD19 CAR T-cell, based on phase II outcomes for 75 pediatric and young adult patients treated in a multicenter international study. ${ }^{53}$

A Phase I/IIA study evaluated the therapy in 30 ALL patients (pediatric and adults); 22 patients had two or more 
relapses and only three patients had one ALL relapse. A complete remission was seen in $90 \%$ (27 of 30) of the patients. A remarkable finding from this study was a high remission rate $(60 \%)$ in patients in whom allogeneic stem cell transplantation had failed. Moreover, among the enrolled patients, there were three patients who had progressed on blinatumomab, thus suggesting that CD19targeting CAR-T therapy is a promising strategy, even for the patients who have progressed on other CD19targeting therapies. ${ }^{52}$

Another multi-center, single-cohort, phase II (ELIANA trial) reported data in 75 pediatric patients. All patients, with a median age of 11 years and a history of one to eight previous therapies, received tisagenlecleucel. The overall remission rate was $81 \%$ with the six-month relapse-free survival rate and overall survival rates being $80 \%$ and $90 \%$, respectively. The relapse-free survival rate was $59 \%$ at one year, and CAR-Ts were reported to persist in the blood for as long as 20 months. A single tisagenlecleucel infusion was found to result in highly efficient and durable remission in children and young adults in this global study. ${ }^{53}$

These encouraging data and due to the universal expression of CD22 in B-cell ALL cells, new strategies to improve CAR $\mathrm{T}$ cell outcomes may include CAR-T cells directed against CD22, 55 and CD19/CD22 dual-targeted constructs. ${ }^{56,57}$

In conclusion, CAR T-cell is a promising therapy with a high response rate, mainly in patients with relapsed/ refractory CD19+ B-cell ALL after two prior lines of therapy or refractory to first-line therapy in patients up to 25 years of age. In addition, the data suggest that CAR-T therapy might still be an important therapy for patients who have progressed on other CD19-targeting therapies. With greater experience using CAR T cell therapy, it is hoped that the attendant toxicities will be ameliorated such that future consideration may be given to incorporate CAR-T therapy earlier in the course of high-risk disease, such as persistence of MRD-positivity.

\section{Summary and Conclusion}

The time-honored primary therapy of ALL has as its backbone steroids and chemotherapy. The morbidity and mortality from induction were primarily related to opportunistic infections (eg, aspergillosis) in the post-chemotherapy neutropenic setting. The prognosis of patients who failed to enter an initial CR, or MRD-negative CR, as well as those who relapsed, was almost universally fatal. The advent of targeted monoclonal antibody therapy allowed for the first time to convert an MRD-positive CR to MRD-negativity and increase the survival of patients. Similarly, patients who relapsed could use monoclonal-antibody therapy as a bridge to an allogeneic HSCT as a curative modality. Furthermore, the advent of chimeric antigen receptor (CAR)-T has allowed, for the first time, to offer realistic hope also to patients with truly advanced disease. The challenge of the coming decade is to more precisely define the role of monoclonal antibody therapy, reduce the attendant toxicities of CAR-T therapy, and ultimately bring all these novel approaches to the upfront setting. In time, the hope is to bring the cure rate of adult ALL closer to the remarkable feat achieved in childhood ALL.

\section{Acknowledgment}

The authors wish to gratefully acknowledge the assistance of Beth Zisman in the preparation of the manuscript.

\section{Disclosure}

The authors report no conflicts of interest in this work.

\section{References}

1. Paul S, Kantarjian H, Jabbour EJ. Adult acute lymphoblastic leukemia. Mayo Clin Proc. 2016;91(11):1645-1666. doi:10.1016/j. mayocp.2016.09.010

2. Samra B, Jabbour E, Ravandi F, et al. Evolving therapy of adult acute lymphoblastic leukemia: state-of-the-art treatment and future directions. J Hematol Oncol. 2020;13(1):70. doi:10.1186/s13045020-00905-2

3. Czuczman MS, Olejniczak S, Gowda A, et al. Acquirement of rituximab resistance in lymphoma cell lines is associated with both global $\mathrm{CD} 20$ gene and protein down-regulation regulated at the pretranscriptional and posttranscriptional levels. Clin Cancer Rec. 2008;14 (5):1561-1570.

4. Piccaluga PP, Arpinati M, Candoni A, et al. Surface antigens analysis reveals significant expression of candidate targets for immunotherapy in adult acute lymphoid leukemia. Leuk Lymphoma. 2011;52 (2):325-327. doi:10.3109/10428194.2010.529206

5. Hoelzer D, Walewski J, Döhner H, et al. Improved outcome of adult Burkitt lymphoma/leukemia with rituximab and chemotherapy: report of a large prospective multicenter trial. Blood. 2014;124 (26):3870-3879. doi:10.1182/blood-2014-03-563627

6. Thomas DA, O'Brien S, Faderl S, et al. Chemoimmunotherapy with a modified hyper-CVAD and rituximab regimen improves outcome in de novo Philadelphia chromosome-negative precursor B-lineage acute lymphoblastic leukemia. J Clin Oncol. 2010;28(24):3880-3889. doi:10.1200/JCO.2009.26.9456

7. Hoelzer D, Huettmann A, Kaul F, et al. Immunochemotherapy with rituximab improves molecular $\mathrm{CR}$ rate and outcome in $\mathrm{CD} 20$ B-lineage standard and high-risk patients; results of $263 \mathrm{CD} 20$ patients studied prospectively in GMALL study 07/2003. Blood. 2010;116 (21):170. doi:10.1182/blood.V116.21.170.170

8. Maury S, Chevert S, Thomas X, et al. Rituximab in B-lineage adult acute lymphoblastic leukemia. $N$ Engl $J$ Med. 2016;375 (11):1044-1053. doi:10.1056/NEJMoa1605085 
9. Cheson BD. Ofatumumab, a novel anti-CD20 monoclonal antibody for the treatment of B-cell malignancies. J Clin Oncol. 2010;28 (21):3525-3530. doi:10.1200/JCO.2010.27.9836

10. Jabbour E, Richard-Carpentier G, Sasaki Y, et al. Hyper-CVAD regimen in combination with ofatumumab as frontline therapy for adults with Philadelphia chromosome-negative B-cell acute lymphoblastic leukaemia: a single-arm, Phase 2 trial. Lancet Haematol. 2020;7(7): e523-e533. doi:10.1016/S2352-3026(20)30144-7

11. Shah NN, Stevenson MS, Yuan CM, et al. Characterization of CD22 expression in acute lymphoblastic leukemia. Pediatr Blood Cancer. 2015;62(6):964-969. doi:10.1002/pbc.25410

12. Raetz EA, Cairo MS, Borowitz MJ, et al. Chemoimmunotherapy reinduction with epratuzumab in children with acute lymphoblastic leukemia in marrow relapse: a children's oncology group pilot study. $J \quad$ Clin Oncol. 2008;26(22):3756-3762. doi:10.1200/JCO.20 07.15.3528

13. Raetz EA, Cairo MS, Borowitz MJ, et al. Re-induction chemoimmunotherapy with epratuzumab in relapsed acute lymphoblastic leukemia (ALL): phase II results from Children's Oncology Group (COG) study ADVL04P2. Pediatr Blood Cancer. 2015;62(7):1171-1175. doi:10.1002/pbc.25454

14. Advani A, McDonough S, Coutre S, et al. Southwest Oncology Group Study S0910: a phase 2 trial of clofarabine/cytarabine/epratuzumab for relapsed/refractory acute lymphocytic leukemia. $\mathrm{Br}$ J Haematol. 2014;165(4):504-509. doi:10.1111/bjh.12778

15. Keyhani A, Huh YO, Jendiroba D, et al. Increased CD38 expression is associated with favorable prognosis in adult acute leukemia. Leuk Res. 2002;24(2):153-159. doi:10.1016/S0145-2126(99)00147-2

16. Mirgh S, Ahmed R, Agrawal N. Will Daratumumab be the next game changer in early thymic precursor-acute lymphoblastic leukaemia? $\mathrm{Br}$ J Haematol. 2019;187(2):e33-e35. doi:10.1111/bjh.16154

17. Krejcik J, Casneuf T, Nijhof IS, et al. Daratumumab depletes CD38+ immune regulatory cells, promotes T-cell expansion, and skews T-cell repertoire in multiple myeloma. Blood. 2016;128(3):384-394. doi:10.1182/blood-2015-12-687749

18. Bride KL, Vincent TL, Im SY, et al. Preclinical efficacy of daratumumab in T cell acute lymphoblastic leukemia. Blood. 2018;131 (9):995-999. doi:10.1182/blood-2017-07-794214

19. Bonda A, Punatar S. Daratumumab at the frontiers of post-transplant refractory $\mathrm{T}$ acute lymphoblastic leukemia-a worthwhile strategy? Bone Marrow Transplant. 2018;53(11):1487-1489. doi:10.1038/ s41409-018-0222-5

20. Ofran Y, Ringelstein-Harlev S, Slouzkey I, et al. Daratumumab for eradication of minimal residual disease in high-risk advanced relapse of T-cell/CD19/CD22-negative acute lymphoblastic leukemia. Leukemia. 2020;34(1):293-295. doi:10.1038/s41375-019-0548-z

21. Hu Y, Turner MJ, Shields J, et al. Investigation of the mechanism of action of alemtuzumab in a human CD52 transgenic mouse model. Immunology. 2009;128(2):260-270. doi:10.1111/j.1365-2567.2009.0 3115.x

22. Angiolillo AL, Yu AL, Reaman G, et al. A phase II study of Campath-1H in children with relapsed or refractory acute lymphoblastic leukemia: a Children's Oncology Group report. Pediatr Blood Cancer. 2009;53(6):978-983. doi:10.1002/pbc.22209

23. Stock W, Sanford B, Lozanski G, et al. Alemtuzumab can be incorporated into front-line therapy of adult acute lymphoblastic leukemia (ALL): final phase I results of a Cancer and Leukemia Group B Study (CALGB 10102). Blood. 2009;114(22):838. doi:10.1182/blood. V114.22.838.838

24. Silva A, Laranjeira ABA, Martins LR, et al. IL-7 contributes to the progression of human T-cell acute lymphoblastic leukemias. Cancer Res. 2011;71(14):4780-4789. doi:10.1158/0008-5472.CAN-10-3606

25. Canté-Barrett K, Spijkers-Hagelstein JAP, Buijs-Gladdines JGCAM, et al. MEK and PI3K-AKT inhibitors synergistically block activated IL7 receptor signaling in T-cell acute lymphoblastic leukemia. Leukemia. 2016;30(9):1832-1843. doi:10.1038/leu.2016.83
26. Akkapeddi P, Fragoso R, Hixon JA, et al. A fully human anti-IL-7R $\alpha$ antibody promotes antitumor activity against T-cell acute lymphoblastic leukemia. Leukemia. 2019;33(9):2155-2168. doi:10.1038/ s41375-019-0434-8

27. Bargou R, Leo E, Zugmaier G, et al. Tumor regression in cancer patients by very low doses of a T cell engaging antibody. Science. 2008;321(5891):974-977. doi:10.1126/science.1158545

28. Topp MS, Gokburger N, Stein AS, et al. Phase II trial of the anti-CD19 bispecific T cell-engager blinatumomab shows hematologic and molecular remissions in patients with relapsed or refractory B-precursor acute lymphoblastic leukemia. J Clin Oncol. 2014;32 (37):4134-4140. doi:10.1200/JCO.2014.56.3247

29. Topp MS, Kufer P, Gokbuget N, et al. Targeted therapy with the T-cell-engaging antibody blinatumomab of chemotherapy-refractory minimal residual disease in B-lineage acute lymphoblastic leukemia patients results in high response rate and prolonged leukemia-free survival. J Clin Oncol. 2011;29(18):2493-2498. doi:10.1200/ JCO.2010.32.7270

30. Himabukuro-Vornhagen A, Gödel P, Subklewe M, et al. Cytokine release syndrome. J Iimmunother Cancer. 2018;56:6.

31. Topp MS, Gokbuget N, Stein A, et al. Safety and activity of blinatumomab for adult patients with relapsed or refractory B-precursor acute lymphoblastic leukaemia: a multicenter, single-arm, phase 2 study. Lancet Oncol. 2015;16(1):57-66. doi:10.1016/S1470-2045(14) 71170-2

32. Caimi PF, Ahmed N, Rojas P, et al. Prophylactic tocilizumab before CD3/4-1bb anti-CD19 car-T cell infusion decreases incidence of severe crs without increased risk of neurotoxicity. Cytotherapy. 2020;22(5):S16-S17.

33. Kantarjan H, Stein A, Gokbuget N, et al. Blinatumomab versus chemotherapy for advanced acute lymphoblastic leukemia. $N$ Engl $J$ Med. 2017;376(9):836-847. doi:10.1056/NEJMoa1609783

34. Foa R, Bassan R, Vitale A, et al. Dasatinib-blinatumomab for ph-positive acute lymphoblastic leukemia in adults. $N$ Engl $\mathrm{J} \mathrm{Med}$. 2020;383(17):1613-1623. doi:10.1056/NEJMoa2016272

35. Kantarjian HM, De Angelo DJ, Stelljes M, et al. Inotuzumab ozogamicin for acute lymphoblastic leukemia. $N$ Engl J Med. 2016;375 (8):740-753. doi:10.1056/NEJMoa1509277

36. Kantarjian HM, De Angelo DJ, Stelljes M, et al. Inotuzumab ozogamincin versus standard of care in relapsed or refractory acute lymphoblastic leukemia: final report and long-term survival follow-up from the randomized, Phase 3 INO-VATE study. Cancer. 2019;125 (14):2474-2487. doi:10.1002/cncr.32116

37. Jabbour E, Ravandi F, Kebriael P, et al. Salvage chemoimmunotherapy with inotuzumab ozogamicin combined with mini-hyper-CVD for patients with relapsed or refractory Philadelphia chromosome-negative acute lymphoblastic leukemia: a phase 2 clinical trial. JAMA Oncol. 2018;4(2):230-234. doi:10.1001/ jamaoncol.2017.2380

38. Sasaki K, Kantarijan HM, Ravandi F, et al. Sequential combination of low-intensity chemotherapy (mini-hyper-CVD) plus inotuzumab ozogamicin with or without blinatumomab in patients with relapsed/ refractory Philadelphia chromosome-negative acute lymphoblastic leukemia (ALL): a phase 2 trial. Blood. 2019;132(Supplement 1):553. doi:10.1182/blood-2018-99-115162

39. Kantarjian HM, Ravandi F, Short NJ, et al. Inotuzumab ozogamicin in combination with low-intensity chemotherapy for older patients with Philadelphia chromosome-negative acute lymphoblastic leukaemia: a single-arm, phase 2 study. Lancet Oncol. 2018;19(2):240-248. doi:10.1016/S1470-2045(18)30011-1

40. Roberts AW, Davids MS, Pagel JM, et al. Targeting BCL2 with venetoclax in relapsed chronic lymphocytic leukemia. $N$ Engl J Med. 2016;374(4):311-322. doi:10.1056/NEJMoa1513257

41. DiNardo CD, Pratz K, Pullarkat V, et al. Venetoclax combined with decitabine or azacitidine in treatment-naive, elderly patients with acute myeloid leukemia. Blood. 2018. doi:10.1182/blood-2018-08-868752 
42. Jain N, Lamb AV, O'Brien S, et al. Early T-cell precursor acute lymphoblastic leukemia/lymphoma (ETP-ALL/LBL) in adolescents and adults: a high-risk subtype. Blood. 2016;127(15):1863-1869. doi:10.1182/blood-2015-08-661702

43. Chonghaile TN, Roderick JE, Glenfield C, et al. Maturation stage of T-cell acute lymphoblastic leukemia determines BCL-2 versus BCL-XL dependence and sensitivity to ABT-199. Cancer Discov. 2014;4(9):1074-1087. doi:10.1158/2159-8290.CD-14-0353

44. Richard-Carpentier G, Jabbour E, Short NJ, et al. Clinical experience with venetoclax in combination with chemotherapy for relapsed or refractory t-cell acute lymphoblastic leukemia.. Clin Lymphoma Myeloma Leuk. 2020;20(4):212-218. doi:10.1016/j.clml.2019.09.608

45. Lacayo NJ, Pullarkat VA, Stock W, et al. Safety and efficacy of venetoclax in combination with navitoclax in adult and pediatric relapsed/refractory acute lymphoblastic leukemia and lymphoblastic lymphoma. Blood. 2019;134.

46. Frey NV, Porter DL. Cytokine release syndrome with novel therapeutics for acute lymphoblastic leukemia. Am J Hematol. 2019;94 (S1):S24-S27. doi:10.1002/ajh.25442

47. Brentjens RJ, Santos E, Nikhamin Y, et al. Genetically targeted $\mathrm{T}$ cells eradicate systemic acute lymphoblastic leukemia xenografts. Clin Cancer Res. 2007;13(18):5426-5435. doi:10.1158/1078-0432. CCR-07-0674

48. Lee DW, Kochenderfer JN, Stetler-Stevenson M, et al. T cells expressing CD19 chimeric antigen receptors for acute lymphoblastic leukaemia in children and young adults: a Phase 1 dose-escalation trial. Lancet. 2015;385(9967):517-528.

49. Davila ML, Riviere I, Wang X, et al. Efficacy and toxicity management of 19-28z CAR T cell therapy in B cell acute lymphoblastic leukemia. Sci Transl Med. 2014;6(224):224ra25
50. Teachey DT, Lacey SF, Shaw PA, et al. Identification of predictive biomarkers for cytokine release syndrome after chimeric antigen receptor T-cell therapy for acute lymphoblastic leukemia. Cancer Discov. 2016;6(6):664-679. doi:10.1158/2159-8290.CD-16-0040

51. Turtle CJ, Hanafi LA, Berger C, et al. CD19 CAR-T cells of defined CD4+: CD8+composition in adult B cell ALL patients. J Clin Invest. 2016;126(6):2123-2138. doi:10.1172/JCI85309

52. Maude SL, Frey N, Shaw PA, et al. Chimeric antigen receptor T cells for sustained remissions in leukemia. $N$ Engl J Med. 2014;371 (16):1507-1517. doi:10.1056/NEJMoa1407222

53. Maude SL, Laetsch TW, Buechner J, et al. Tisagenlecleucel in children and young adults with B-cell lymphoblastic leukemia. $N$ Engl J Med. 2018;378(5):439-448. doi:10.1056/NEJMoa1709866

54. Hunter BD, Jacobson CA. Car t-cell associated neurotoxicity: mechanisms, clinicopathologic correlates, and future directions. J Natl Cancer Inst. 2019;111(7):646-654. doi:10.1093/jnci/djz017

55. Fry TJ, Shah NN, Orentas RJ, et al. CD22-targeted CAR T cells induce remission in B-ALL that is naive or resistant to CD19-targeted CAR immunotherapy. Nat Med. 2018;24(1):20-28. doi:10.1038/ nm.4441

56. Amrolia PJ, Wynn R, Hough RE, et al. Phase I study of AUTO3, a bicistronic chimeric antigen receptor (CAR) T-cell therapy targeting CD19 and CD22, in pediatric patients with relapsed/refractory Bcell acute lymphoblastic leukemia (r/r B-ALL): Amelia Study. Blood. 2019;134(Supplement_1):2620. doi:10.1182/blood-2019-123424

57. Schultz LM, Muffly LS, Spiegel JY, et al. Phase I trial using CD19/ CD22 bispecific CAR T cells in pediatric and adult acute lymphoblastic leukemia (ALL). Blood. 2019;134(Supplement_1):744. doi:10.1182/blood-2019-129411

\section{Publish your work in this journal}

Biologics: Targets and Therapy is an international, peer-reviewed journal focusing on the patho-physiological rationale for and clinical application of Biologic agents in the management of autoimmune diseases, cancers or other pathologies where a molecular target can be identified. This journal is indexed on PubMed Central, CAS, EMBase,
Scopus and the Elsevier Bibliographic databases. The manuscript management system is completely online and includes a very quick and fair peer-review system, which is all easy to use. Visit $\mathrm{http}: / / \mathrm{www}$.dovepress.com/testimonials.php to read real quotes from published authors. 